Magnetic anisotropy and its microstructural origin in epitaxially grown SmCo thin films

\author{
Mohamed Benaissa and Kannan M. Krishnan
}

National Center for Electron Microscopy; Materials Sciences Division, 72-150

Lawrence Berkeley National Laboratory, Berkeley, CA 94720

\author{
Eric E. Fullerton ${ }^{\ddagger}$ and J.S. Jiang
}

Materials Science Division, Argonne National Laboratory, Argonne, IL 60439

\begin{abstract}
The submitted manuscript has been created by the University of Chicago as Operator of Argonne National Laboratory ("Argonne") under Contract No. W-31-109-ENG-38 with the U.S. Department of Energy. The U.S. Government retains for itself, and others acting on its behalf, a paid-up, nonexclusive, irrevocable woridwide license in said article to reproduce, prepare derivative works, distribute copies to the public, and perform publicly and display publicly, by or on behalf of the Government.
\end{abstract}

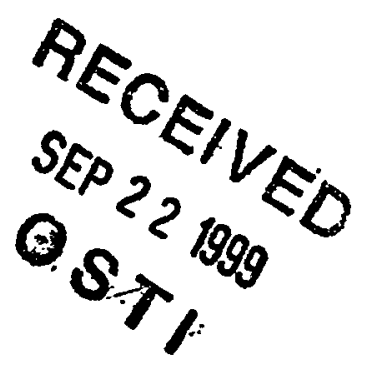

Work supported by the U.S. Department of Energy, Division of Advanced Energy Projects, and the Center for Excellence in Synthesis and Processing, under contract No. DE-AC03-76SF00098 and at Argonne by the US-DOE Division of Basic Energy Sciences-Material Sciences under contract No. W31-109-ENG-38.

On leave from National Institute for Nuclear Res. (ININ), Materials Science Department, Mexico. ${ }^{\ddagger}$ Present address: IBM Almaden Research Center, 650 Harry Rd., San Jose, CA 95120. 


\section{DISCLAIMER}

This report was prepared as an account of work sponsored by an agency of the United States Government. Neither the United States Government nor any agency thereof, nor any of their employees, make any warranty, express or implied, or assumes any legal liability or responsibility for the accuracy, completeness, or usefulness of any information, apparatus, product, or process disclosed, or represents that its use would not infringe privately owned rights. Reference herein to any specific commercial product, process, or service by trade name, trademark, manufacturer, or otherwise does not necessarily constitute or imply its endorsement, recommendation, or favoring by the United States Government or any agency thereof. The views and opinions of authors expressed herein do not necessarily state or reflect those of the United States Government or any agency thereof. 


\section{DISCLAIMER}

Portions of this document may be illegible in electronic image products. Images are produced from the best available original document. 


\title{
Magnetic anisotropy and its microstructural origin in epitaxially grown SmCo thin films
}

\author{
.Hohamed Benaissa" and Kannan :K.Krishnan" \\ . Vutional Center for Electron .Wicroscopy. .Haterials Sciences Division. 72-150. Lawrence Berkeley National Laboraton: Berkeley. \\ CA $9+720$ \\ Eric E.Fullerton ${ }^{-}$and J.S.Jiang \\ .Haterials Sciences Division. Argonne vational Laboratory. Argonne. IL 60)+39-4.9\$5
}

\begin{abstract}
Microstructural features and magnetic behavior of epitaxially grown SmCo thin films with very high in-plane anisotropy are presented. Transmission electron microscopy was used to characterize the microstructure while magnetic measurements were performed using $d c$ and SQUID magnetometers. Two substrate orientations were studied. i.e., $\mathrm{MgO}(100) / \mathrm{Cr}(100) / \mathrm{SmCo}(11 \overline{2} 0)$ and $\mathrm{MgO}(110) / \mathrm{Cr}(211) / \mathrm{SmCo}(1 \overline{1} 00)$. In the former, the $\mathrm{SmCo}(11 \overline{2} 0)$ film shows a bicrystalline microstructure, whereas in the latter, a uniaxial one is observed. Both microstructures consist of grains with a mixture of $\mathrm{SmCo}_{3}, \mathrm{Sm}_{2} \mathrm{Co}_{7}$ and $\mathrm{SmCo}_{3}$ polytypoids. A deviation from the c-axes was observed in the in-plane anisotropy of the $\mathrm{SmCo}(11 \overrightarrow{20})$ thin film. A strong exchange interaction between the grains would, in principle, explain the observed deviation. On the other hand, both $\mathrm{SmCo}(1+\overline{2} 0)$ and $(1 \overline{1} \mathrm{co})$ thin films show very high coercivity values with pinning-type characteristics. Possible coercivity mechanisms related to intergranular exchange interactions and local variation in magnetocrystalline anisotropy constants are discussed.
\end{abstract}

Index Terms- anisotropy. coercivity, epitaxy, hard magnet, microstructure. pinning, thin film, samarium-cobalt.

\section{INTRODLCTION}

Microstructural features such as grain size and shape. phase distribution, grain boundary type, and the mutual crystallographic orientation between grains are parameters that determine the magnetic behavior of modern permanent magnets. Such permanent magnets, generally composed of rare-earth-based compounds. are characterized by high coercivity and energy product. Nowadays. high coercivity values $\left(\mathrm{H}_{\mathrm{c}}=+T\right.$ ) are routinely obtained in bulk hard magnetic materials such as SmCo or NdFeB-based alloys. In the case of thin tilms. such pertiormance was not reached in practice. fundamentally because of insufficient control of

\footnotetext{
- On leave from National Institute for Nuclear Res. (ININ). Materials Sitence Dept.. Mlexico. e-mail mohamed e fenix.itisicacu.unam.mx

$\approx$ To whom correspondence should ie addressed. $(510)+36+614$. Fax 1510)+865888. c-mail krishnan@ibl.gov

- Present address: IBM .Almaden Research Center. 650 Harty Road. San Jose C.t 95120

This work was supported by the Li.S. Department of Energy. Disision of Advanced Energy Projects. and the Cencer of Excellence in Synthesis and Processing. under contract. To. DE-ACO:- 6 SFO0003.

Wiork at $A$. VL was supported by the $L$ 's DOE. BES-Mtarerials Stiences. under Contrate .No. W-31-1119-E.YiG-is
}

microstructural parameters. Early attempts to grow SmCo in the form of thin films generated large coercivity values [1], although a clear explanation for such an improvement in coercivity torces was not presented. However, it has recently been demonstrated that growth on selective substrates with appropriate surface orientation and temperature. leads to epitaxial Sm-Co thin films with very high coercivity values (comparable to those of bulk hard magnets) resulting from the high in-plane anisotropy [2], [3]. The objective of the present work is to shed light on the microstructural features, such as grains size and their mutual orientation, microstructural imperfection and phase distribution, that induce such a strong. in-plane anisotropy and to relate them to the magnetic behavior of the film.

\section{EXPERIMENTTAL}

Sm-Co thin films were prepared by magnetron sputtering as described in [?] and [3]. Cr buffer layers, $200 \mathrm{~A}$ thick, were deposited onto single-crystal $\mathrm{MgO}(100)$ and (110) substrates held at a temperature. $\mathrm{T}_{s}$ of $600^{\circ} \mathrm{C}$ resulting in $\mathrm{Cr}(100)$ and (211) epitaxial growth. respectively. The Sm-Co films were subsequently deposited with a nominal composition of $\mathrm{Sm}_{2} \mathrm{Co}_{7}$ by co-sputtering from separate $\mathrm{Sm}$ and $\mathrm{Co}$ sources at $T_{i} \equiv 600^{\circ} \mathrm{C}$ and $+\mathrm{m}$ Tor Ar pressure to a thickness of about $750 \AA$. The Sm-Co thin tilms were then capped by a thin (50 A) $\mathrm{Cr}$ layer. For plan view transmission electron microscopy (TEM) and microanalyses. disks of three millimeters diameter were prepared, dimpled and ion milled to perforation. The average and local chemical composition of the films were measured using energy dispersive $x$-ray spectroscopy (EDXS) in an analytical TEM (JEOL 200CX), while electron diffraction and high-resolution TEM (HRTEM) imaging were performed using a Topcon 002B microscope operated at 200 $\mathrm{keV}$. The magnetic properties were measured with a Quantum Design de magnetometer equipped with a 9-T magnet and a 7 $T$ SQUTD magnetometer equipped with both longitudinal and transverse coils.

\section{RESLLTS AND DISCLSSION}

The crystal structure of $\mathrm{Cr}$ is body centered cubic (bec) whilst SmCo alloys are generally either hexagonal or rhombohedral. However. when deposited at elevated substrate temperature on $\mathrm{MgO}$. Cr establishes an epitaxial relationship with the substrate providing a template for the subsequent growth of the SmCo film. Fig.I shows the microstructure of the as-yrown SmCo thin films. For MgO(100) substrates. 


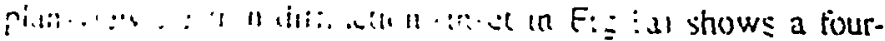

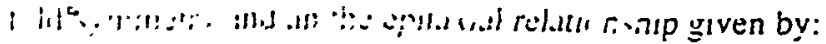

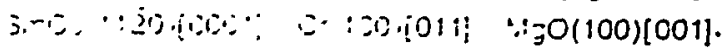

whle in the ...

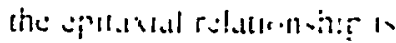

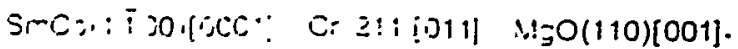

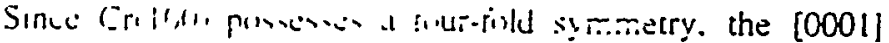

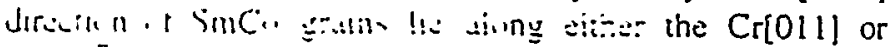
Cr:01 $\bar{i}$ irs

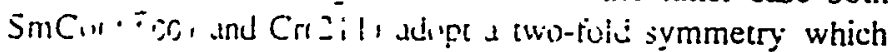
rauls in a uniakial verisiure , Fis.lb).

In the eisc of MgOilon, substrate (Fig.la) the SmCin 1120 , rilm ministructure $=$ xhibits small grains $(-25$ 60) $\mathrm{nm}$ in w/s, with this!r i-.txes normal ar parallel to each other wulung in a hi:-s italline structure. where the grains are sepursted h! incinerent tus bounbar:s. In the case of MgOIllill shirate. the mirusirditure mataly shows grains $1-50 \mathrm{~nm}$ in size, haing their :-axes parallel to each other resulting in a uniaxial structure. Stacking disorder, normal to the c-axis within the grain structure, can be observed in the micrographs (Fig. 1). This stacking disorder can be described as an intergrowth of difierent polytypoids of SmCo alloys. Indeed. vlose examination using HRTE.M (Fig.2), combined with image simulations and measurements of $d_{(x)) !}$ spacings, shous that the stakking disorder within a given grain correspunds to the dirterence in stacking cetween $\mathrm{SmCo}_{3}$. $\mathrm{Sm}: \mathrm{Co}$ - and $\mathrm{SmCo}$; phases. The micrograph shows that these SmCo polytypoids ha: a a common c-axis. i.e.. their basal planes are parallel to each other. The crystal structures of

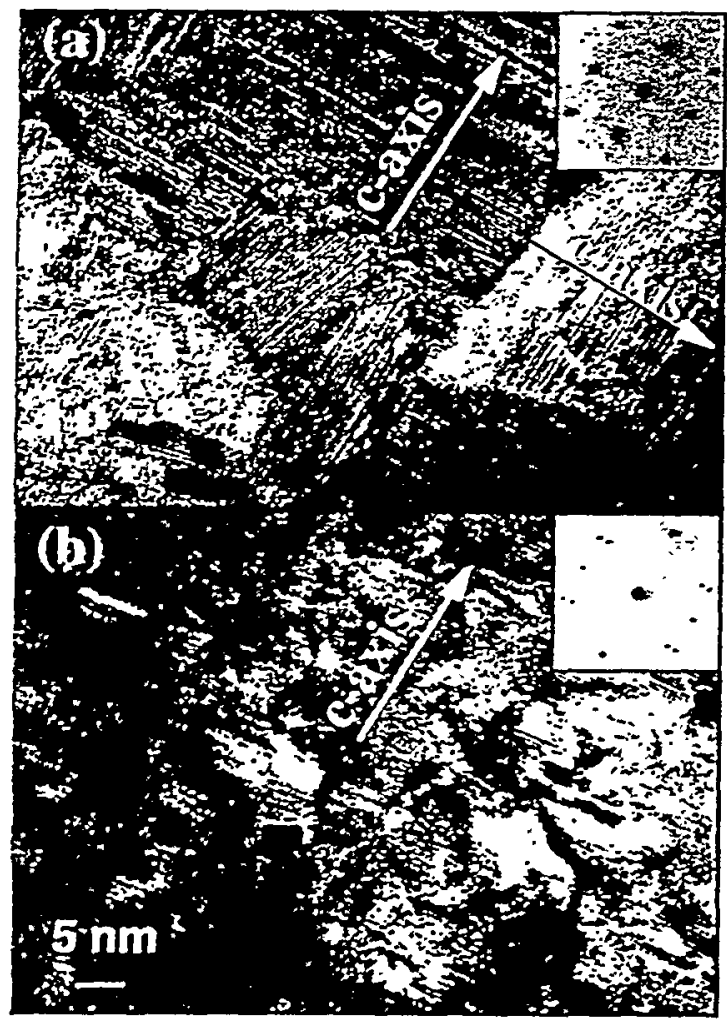

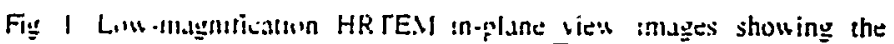

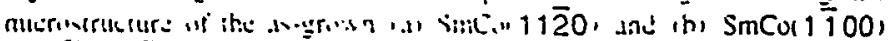

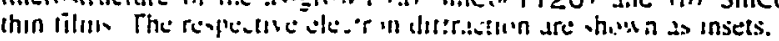

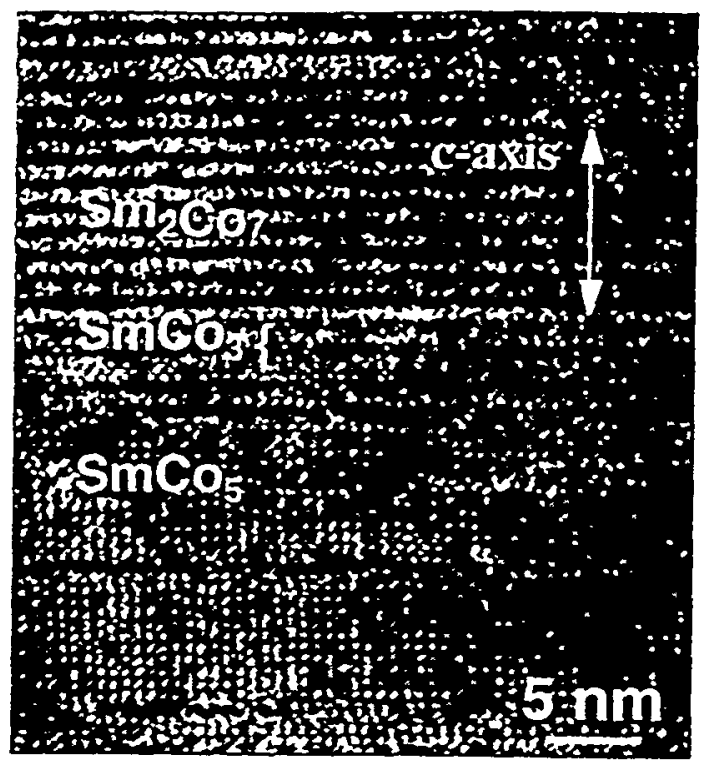

Fig.2. HRTEM in-plane view image of an individual grain indicating the stacking disorder along the c-axis. or the stacking of different SmCo polytypoids with a common c-axis.

intermetallic Sm-Co phases. ranging from $\mathrm{SmCo}_{2}$ to $\mathrm{SmCo}_{3}$ - are closely related and are based on a regular stacking along the c-axis of two kinds of layers. One layer is of the SmCo. Laves phase type and the other is that of $\mathrm{SmCo}_{5}$ type. Since each bilayer is Co-rich. removing one bilayer from $\mathrm{Sm}_{-} \mathrm{Co}_{7}$ for instance. results in the formation of $\mathrm{SmCo}_{\text {s }}$ stacking type. Furthermore. our chemical microanalyses over either an ensemble of grains or a single grain showed that the thin film contained $\mathrm{Sm}$ and $\mathrm{Co}$ in an atomic ratio corresponding to the formula $\mathrm{Sm}_{1,2 .} \mathrm{Co}_{1,26}$, a concentration that is relatively close to the nominal value. within the measurement error. On the other hand. the binary phase diagram of $\mathrm{Sm}-\mathrm{Co}[4]$ shows that a small excess of $\mathrm{Sm}(\mathrm{Co})$ would push the $\mathrm{Sm}_{2} \mathrm{Co}_{\text {; }}$ phase towards an equilibrium with its adjacent compound $\mathrm{SmCo}_{3}\left(\mathrm{SmCO}_{3}\right)$. In summary. the growth of $\mathrm{Sm}_{-} \mathrm{Co}_{\text {; }}$ thin tilms can be described as initiated by an island growth-mode. as indicated by atomic torce microsiop: images [2]. [3], with the basal planes normal to the film surtiace. Local departure from the nominal stoichiometry may occur during the growth. forming regions with either excess $\mathrm{Sm}$ resulting in the formation of $\mathrm{SmCO}_{3}$ or excess $\mathrm{Co}$ leading to the formation of $\mathrm{SmCo}_{\text {. }}$. To minimize the elastic strain energy. the basal planes of the three phases are driven to be parallel to each other along a common c-axis.

Both SmCoi $11 \overline{2} 0)$ and $(1 \overline{1} 00$ ) thin tilms showed very high coercivity values and seemed to indicate characteristics of pinning-type magnets (Fig.3). Particularly in the former case

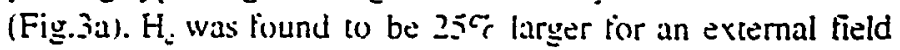
applied parallel to the MIgO[OII] ind $[0 \bar{i} 1]$ directions than that for the one applied alung the . $I g O[0101$ and $[001]$ directions (parallel to the SmC1) c-axes). Due to its bicrystalline microstructure. the SmC(w1120) thin film exhibits a tour-fold symmetry in the in-plane angular dependence of the anisotropy with erfictale calsy axes along the $\mathrm{MlgO}\left[0111\right.$ and $\mathrm{MgO}\left[\mathrm{T}_{1} 1\right]$ dircitiuns. For $\mathrm{MgO}(110)$ 
substrates with $\mathrm{SmCo}(1 \overline{1} 00)$ thin films (Fig.3b), the in-plane angular dependence of the anisotropy shows a two-fold symmetry (or uniaxial) with the c-axis of the SmCo grains (parallel to the $\mathrm{MgO}[001]$ axis) being the easy direction.

The magnetic hardness of SmCo thin films appears to be very sensitive to the crystallography of the phases present as well as the composition and very fine-scale structural details within and at the boundaries of the nanometer-size grains. Two kinds of magnetic interactions exist between neighboring SmCo grains with a given crystallographic orientation. The first is magnetostatic which depends mainly on the shape of the grains, whereas the second, exchange interaction, strongly depends on the grains size. Micromagnetic modeling has shown that for nanometer-scale grains in an isotropic permanent magnet. the exchange interaction between grains is dominant in determining its magnetic behavior [5]. In particular, at the remanent state, the spontaneous magnetic polarization was found to deviate from the easy axes of the grains in the vicinity of the grain boundaries. A similar model, involving strong exchange interactions between the grains in the $\mathrm{Sm}-\mathrm{Co}(11 \overline{20})$ film would, in principle, explain the observed anisotropies (Fig.3). Since the epitaxial growth constrains the c-directions of the $S m-C o$ grains to essentially lie perpendicular (i.e. only two possibilities. in plane) to each other. strong inter-granular exchange interaction would result in the easy direction being at an intermediate orientation. i.e. along $\mathrm{MgO}[011]$ or $\mathrm{M}$ [gO[0-11]. However, if all the SmCo grains were strongly exchange coupled, individual grain boundaries would not be the likely pinning site to explain the pinning-type coercivity mechanism observed in these films. So the question remains: what is really causing the pinning mechanism in these SmCo hard magnet films? Two alternative explanations can be proposed. It has recently been reported that in Co-based alloy
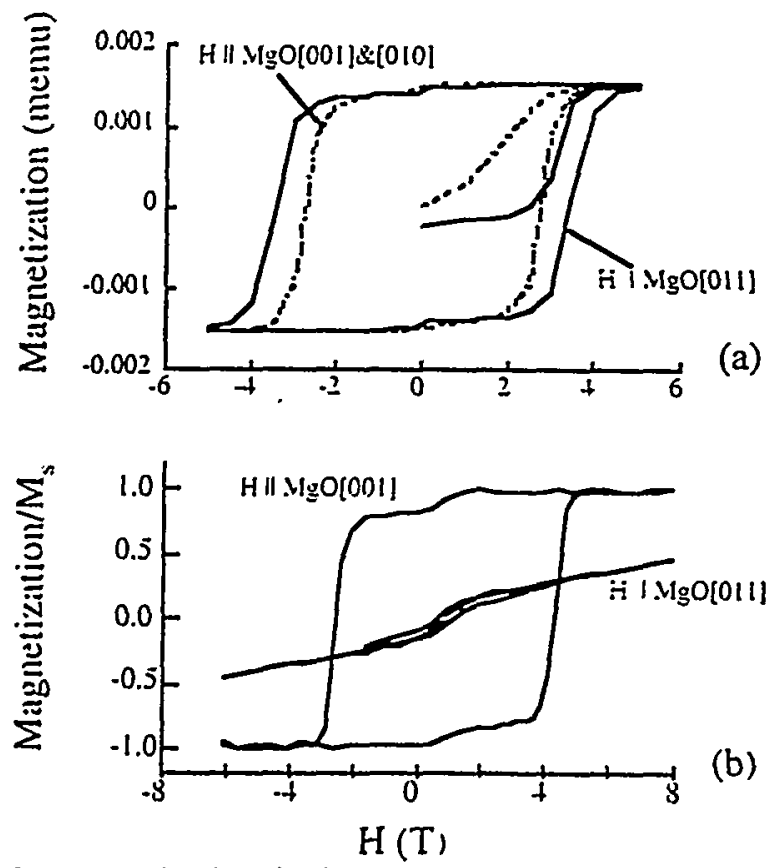

Fig.3. (a) and (b) show in-plane magnetization loops measured along Jifierent axes within the $(11 \overline{2} 0$, and $(1 \overline{100}$ ) films. :spectiveley. Niote the increase in coercivity along. WhOOO(O) I] in (a). thin films with a bicrystalline microstructure [6]. a "cluster" formed by an ensemble of adjacent grains may lead to an effective anisotropy along an intermediate direction and with a magnitude depending on the strength of the inter-granular exchange coupling. One can possibly assume that due to the ferromagnetic exchange coupling between the grains, a

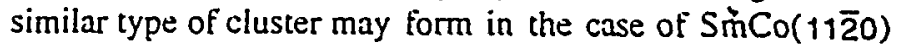
thin films. such that each cluster is considered as a large grain with an effective anisotropy along the $\mathrm{MgO}[011]$ or $\mathrm{MIgO}\left[0_{1} 1\right]$. In such a model. the incoherent twins at the grain boundaries may serve as attractive pinning sites for the magnetic domain walls. The second hypothesis involves the stacking disorder, or the SmCo polytypoids, observed within a given grain with strong variation in local magnetocrystalline anisotropy constants. Although $\mathrm{Sm}_{2} \mathrm{Co}_{7}$ has a relatively high anisotropy [7], that of $\mathrm{SmCO}_{3}$ is much higher and hence, it may impede the movement of the domain walls during the magnetization reversal since it has a larger wall energy. This process is only possible if the domain wall motion is parallel to the c-axis. If the magnetization direction is normal to the c-axis, the energy of the wall is independent of the difference in stacking sequence and should not a priori act as a pinning center.

\section{CONCLUSIONS}

Microstructural features and magnetic behavior of epitaxially grown $\operatorname{SmCo}(11 \overline{2} 0)$ and $\operatorname{SmCo}(1 \overline{1} 00)$ thin films with very high in-plane anisotropy were studied. The $\mathrm{SmCo}(11 \overline{2} 0)$ film showed a bicrystalline microstructure. while the SmCo $(1 \overline{1} 00)$ film showed a uniaxial one. Both microstructures consist of grains with a stacking disorder, or a mixture of $\mathrm{SmCo}_{3}, \mathrm{Sm}_{2} \mathrm{Co}_{7}$ and $\mathrm{SmCo}_{5}$ polytypoids. Although good indications of ferromagnetic interactions between individual SmCo grains were detected. it is still not very clear whether the pinning behavior controlling the coercivity mechanism of the SmCo thin films is due to the incoherent twinning between adjacent grains or to the difference in the magnetocrystalline anisotropy constants of the polytypoids present within the grains. Establishing the validity of either model will involve further studies of the magnetic domain structure in the films. Lorentz microscopy measurements to answer these questions are in progress.

\section{REFERENCES}

[1] H.C.Theuerer. E.A.Vesbitt and D.D.Bacon. "High-coercive-force ma-earth alloys films by getter sputtering" J.Appl.Phy.s.. vol. 10. pp. 2994-2996. June 1969.

[2] E.E.Fullerton et al.. "Structure and magnetism of epitarral rare-earthtransition-metal tilms" Appl.Phys.Letl.. vol. 69. pp. 2488-240. April
1996.

(3) E.E.Fullerton et al.. "High Coercivity. Epitaxial Sm-Co Films with Uniaxial in-plane Anisotropy".4ppl.Phys.Letl.. in press.

[t] Q.G.Wei. H.W.Chang and C.C.Yu. "Reinvestigation of the Sm-Co system". Z.Mkde. vol. \$4. pp. 165-169. 1993.

[5] T.Schreft. J.Fidler, and H.Kronmüller. -Remanence and coercivity in isotropic nanocrystalline permanent magnets". Phys. Ret: B.v vol. +9.

- pp. 6100-6110. Warch 1994.

[6] Q.Peng et al.. "Wlicromagnetic and expenmental studies of CoPrCr polycrystalline thin Hilm media with bicrystal microstructure". IEEE Triuls. Wayn.. vol. 31. pp. 2821-2823. November 1995.

[7] K.H.J.Buschow. "Magnetic anisotropy of some rate earth-cobait compounds $\left(R_{2} C_{0}\right) "$. J. Less-Commmn . Weruls. vol. : $:$ pp. $311-312$. 1973 